\title{
Application and Manifestation of Digital Printing in the Design of Casual Clothing
}

\author{
Longlin Luo \\ South China University of Technology \\ Guangzhou, China
}

\begin{abstract}
With the diversification of society, people are having more and more demands for the personalized clothing, and more and more people are fond of the clothing with unique and novel patterns. Therefore, it is necessary to create a wide variety of new patterns to provide innovative ideas to the development of fashion design. As an emerging printing and dyeing technique, digital printing has gradually showed its own advantages: it is convenient to typeset and design on the computer; the speed of printing and dyeing is faster; resource waste is reduced, which is green and environmentally friendly; and so on. The digital printing is in line with the development direction of socialized large scale production, and it can promote the designers to fully play their inspirations and design diverse patterns. It has been playing an increasingly important role in the fashion trends. Combined with the design theme, based on the main concept of the design expression, the content of the designed patterns is visually displayed in the series of clothing design through the digital printing technique, and the form beauty of the creative clothing is displayed through various clothing styles. In this paper, I mainly explored the specific applications of digital printing in the design of a series of casual wear, including the production of garments, craftsmanship, and creative styling. And I mainly emphasized the influence of digital printing on the use of fabrics, color display, and decorative visual effects of the clothing.
\end{abstract}

\section{Keywords—digital printing; casual wear; pattern design}

\section{INTRODUCTION}

In recent years, the textile printing and dyeing industry is developing rapidly. Digital printing is a processing technique that intuitively conveys the design concept and makes the patterns more vivid. It is widely used in the production of a variety of apparel products. With the production and consumption of digitally printed various textile patterns, people's daily wear will become more lively and vivid. It can meet the needs of consumers in the diversity and environmental friendliness of clothing.

Combined with the current fashion trends, this series of clothing has been designed to be loose and leisure, highlighting the fashion charm of being personalized, young, and lively. The design was inspired by the element of "newspaper", the carrier in which the changes of era have been recorded. As for the processing techniques, the combination of digital printing, hollowing, ripping and splicing is used to imprint the spirit of the new social life in the new era.

In this article, I have explored the different ways in which digital printing is used in fashion design. According to the specific requirements of digital printing for pattern design and fabric application, I have explored the design scheme that can show the best effect of the designed patterns through a variety of trial printings. In this paper, I have studied the specific distribution of the patterned block in the ready-made clothes, the connection and folding between the printed pattern and the style piece, the size and layout of the patterns in the garment, hoping to make the single piece garment not only unique and independent by itself, but also be a natural part of the harmonious whole set of garments.

\section{OVERVIEW OF Digital PRINTING}

\section{A. Operational Principle}

It is the process of digital printing that firstly various drawn patterns are transferred into a computer in their digital forms, then they are processed by the color separation printing system of the inkjet printing machine, and next the printing system spray the dyes onto the corresponding fabrics, finally after a series of processing, colorful patterns can be formed.

\section{B. Features}

The proofing speed of digital printing is faster and the original long process is shortened; and the digital printing has high flexibility to meet people's requirements in orders for goods in a short period of time. The replacement of the original traditional printing machine by digital printing can shorten the original long proofing time, avoid waste of time in production and greatly reduce production costs. Water and color paste are not used in the digital printing process, which greatly reduces waste of resources and pollution to environment, and is consistent with the sustainable development concept. In addition, the fastness values are all high, meeting the requirements of the producers. The automatic order taking is realized through the Internet, and the production process is highly automated. The digitally printed and dyed patterns have high color precision and rich details, and the proofing effect is better through digital printing. 


\section{THE METHODS AND REQUIREMENTS FOR PATTERN DESIGN}

\section{A. Computer Drawing and Type Setting for Pattern Design}

The design of the pattern is inspired by the "newspaper". The outstanding parts in the materials have been extracted and redesigned. The design is based on the color and material of the daily newspaper, and different colors are extracted and combined in the design. The morphological characteristics, colors and concepts expressed through the inspirational object are analyzed to make the theme of the design expressed clearer. After the single pattern design is completed, it is necessary to carry out the two-way continuous and the square continuous connection, and the layout and size of the pattern are set according to the situation of the fabric. The gaps are connected and the complete effect of the seam is adjusted so that a single pattern can be repeatedly duplicated. In this digital printing, the method of first printing and then cutting the piece has been adopted, firstly printing and dyeing the patterns on the solid color base cloth, and then cutting the corresponding pieces according to the patterns (see "Fig. 1").

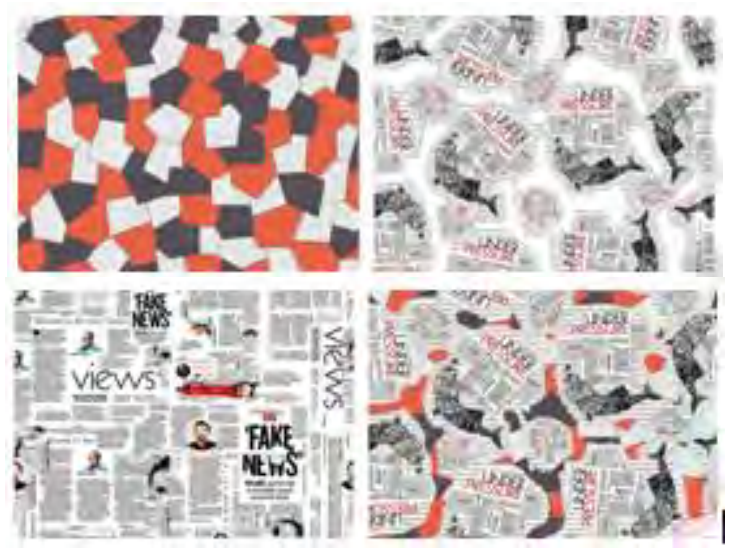

Fig. 1. Computer designs.

\section{B. The Influence of Fabric Properties on the Color Performance of Pattern Design}

In digital printing, the influence of fabric properties on the color performance of the patterns needs to be considered. The color printed on fabrics with different compositions and different textures will present different effects. If the designed pattern is printed on some easily-dyed fabrics, it will present better colors and better color fastness. However, if the designed pattern is printed on cloths that are not easy to absorb color, the colors of it will be relatively light, and the pattern less obvious. Combining with the colors of the pattern designs, this time I have made several trials to print and dye the patterns on the white bottom fabrics.

\section{The SubJect APPlication CONCEPT OF THE Design THEME OF "IMPRINT"}

\section{A. Description of the Theme}

The theme of this series of clothing is inspired by the newspapers people see every day. Newspapers are the readings placed in a corner in people's daily life. As the carrier of popular culture communication, the newspaper plays a decisive role in influencing the public opinions. It is the epitome of people's daily life, the witness of social development and evolution, and represents the brilliant development track of the former paper age. People are familiar with it, but now have felt a bit strange about it Nowadays, people can quickly get news and various information through computers or mobile phones. There is less and less people paying attention to and reading the traditional paper media, and people's lifestyles in the old and new times are completely different from each other due to the development of society. Nowadays, the emergence of new technologies is causing changes to the society. With the advent of the information age, people's lifestyles have undergone great changes, and at the same time, they begin to pursue the concept of individuality and fashion. The new technique of digital printing has been used in this series of garments, and the secondary re-producing designs such as hollowing, splicing, and ripping have been combined with to print out the new look of life and new atmosphere of the times in the garments (see "Fig. 2").

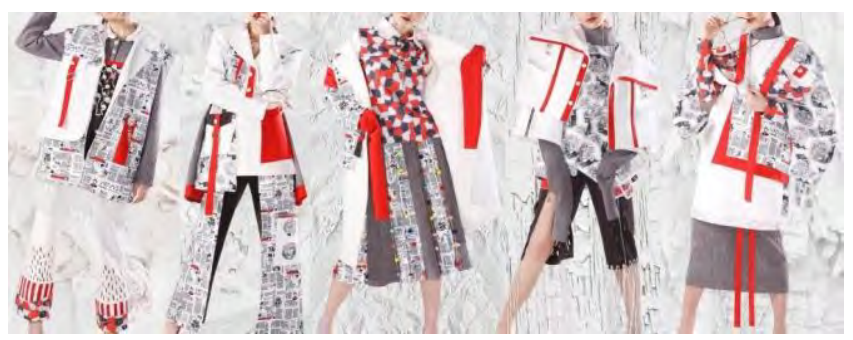

Fig. 2. Display of the series of ready-made garments

\section{B. Analysis of the Inspiration Board}

According to the design ideas, form the associated pictures in mind, and deepen various thinking routes. Develop the design basing on the existing materials and keep capturing the associated contents about the materials. Combine fashion trends and market needs to design the clothing. Taking a certain range of colors as the core color combining with the design theme, and refine the core color of the color card as the main color block. Then analyze the current lifestyle of people to determine the general trend of the clothing silhouette, so that the clothing can be both artistic and practical. People in modern society are fonder of the liberal, dignified, simple and faddish styles (see "Fig. 3"). 

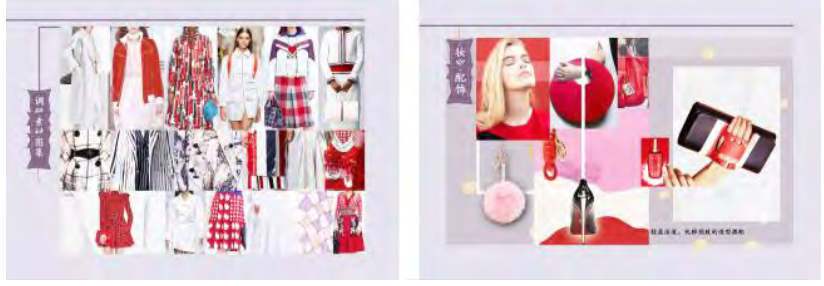

Fig. 3. Concept board of the color trend and style silhouette.

The selected materials are classified according to their types into one inspiration board, and the creative points in the clothing are analyzed and taken as the references for creation, such as the irregular changes of the collar structure, the decorative design of the belt, and the various types of the cuffs. The relationship between the collision and contrast of colors and the coordination of the whole garment should be paid attention to when implementing color matching in the garment. The small decorative parts and functional details of the garment, such as the size, color and stitching of the zipper which can reflect the novelty of the garment, the positions and types of pockets displayed in the garment, and the specific expression of the texture of the garment should all be considered. The accessory design is a relatively important part of the design of the garment. The style of the accessory is determined by the style of the garment. By adding fashionable and unique accessories to the garment, the beauty of the garment can be more strongly expressed, showing the individuality of it. And an atmosphere that can make people feel relaxed and lively can be created (see "Fig. $4 ")$.
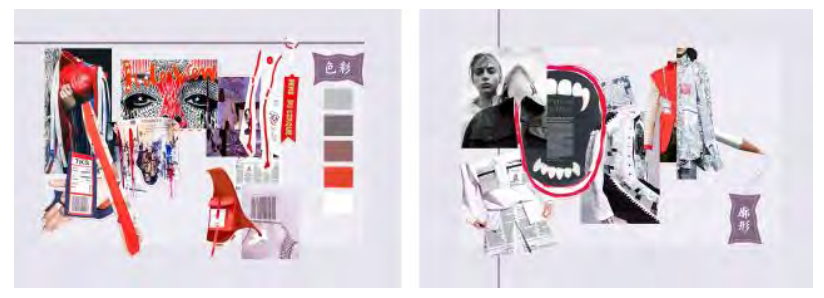

Fig. 4. Concept board of research materials, accessories and makeup.

\section{Renderings, Style Drawings}

Through the combination of text expressions and vivid and interesting graphics, the elements related to newspapers are used as echoes and contrasts to express a new and fashionable wearing concept. Among various colors, red and white are used as the main colors for the garments, and the patterns are digitally printed on the pure white bottom cloth. As for other auxiliary colors, the black, white and grey have been taken into consideration and reflected in the designs, and the sense of coordination and uniformity of the whole series of clothing is formed. In terms of fabrics, the soft knit, comfortable cotton, polyester fiber and other windproof jacket materials are used. At the same time, the limitations of single color and surface of a single garment can be broken through and the colorful appearance of the set of clothing can be achieved through the splicing of different fabrics such as the splicing of the surface with textures and the smooth surface, and through the combination of single-piece clothing.
The style of the clothing is mainly loose and casual. New ideas are added to the details of the clothing, and the accessories are used as detail decorations and embellishments to make the content of the clothes richer. In the matching and design of the accessories, the casual, warm and hearty style of the series of clothing are combined with, and the makeup highlights the warmth, energy and sense of interest. Through the use of this series of elements, the clothing conveys the natural and casual lifestyle of people (see "Fig. 5" and "Fig. 6").

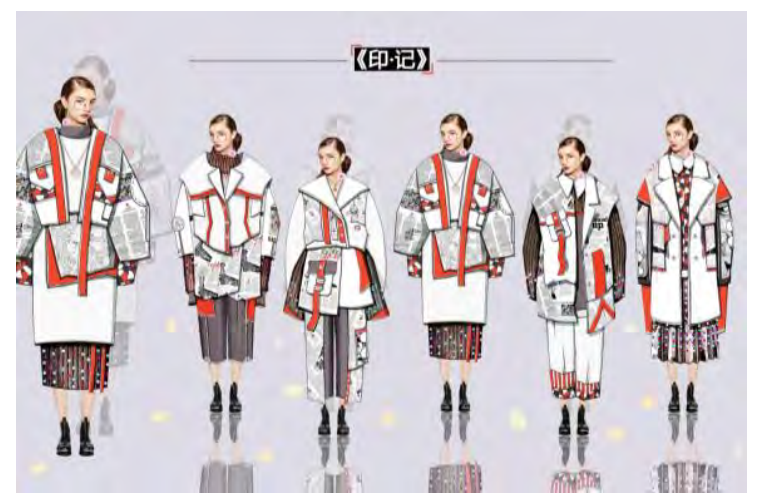

Fig. 5. Renderings.

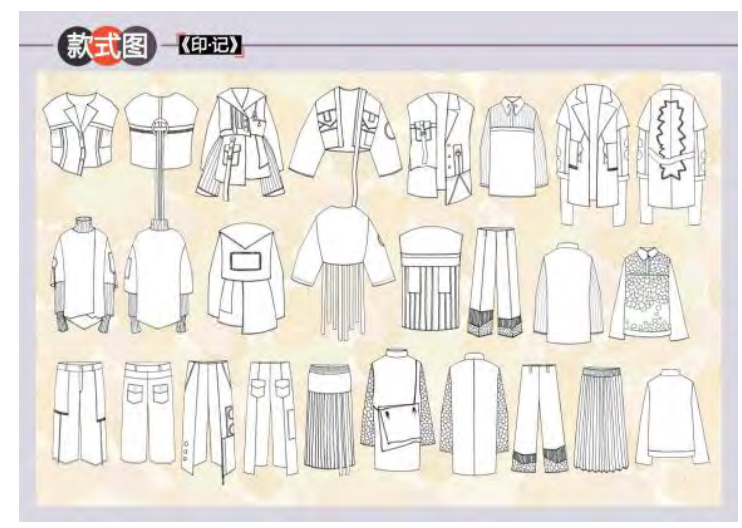

Fig. 6. Style drawings.

\section{The Specific Application of Digital Printing IN THE DESIGN OF THIS SERIES OF CASUAL WEAR}

\section{A. Garment Production}

When producing the garments, the combination of the pattern colors is adjusted according to the overlapping situation of the different patterns. As for the splicing of various parts of the fabric, it should be considered whether the colors have been rationally matched, and the thickness and weight of the fabric will affect the drape state and shape of the garment. Divide the digitally printed fabric into pieces, and calculate the size of the pattern used in the single-piece garment. After collecting the data, the fabric can be cut according to the calculated values, and the elasticity and direction of elasticity of the fabric should be checked before the fabric is cut. Some of the fabrics should be cut according to their directions of yarns, and the performance of the fabric 
determines the overall contour of the garment. During the production process, a white cloth can be used for proofing if necessary, and the general profile of the ready-made garment is estimated according to the sample made of the white cloth. In order to leave enough space for the human body to make various movements, a small amount of space should be reserved in the design when using the elastic fabric, and a relatively large amount of space should be reserved in the design when using the inelastic fabric, which can meet the requirements of people in the degree of comfort of garments.

\section{B. Performance of Techniques}

On the basis of the pieces of the garment with digitally printed patterns, techniques including hollowing, ripping, splicing, and hand-sewn decorative lines are applied for the secondary re-producing design. First, hollowing. Determine the specific part of the piece to be hollowed. Use the pen to gently outline the specific parts and then hollow them one by one, forming the beautiful hollowed piece. Second, ripping. Try a variety of methods, such as selecting a small area to lightly burn the edges, so that the color of this part of fabric will change and be different from the original color. Or the gradual ripping mode of natural extending can be used. Third, splicing. The splicing of the pieces with the digitally printed patterns and the other pieces of the fabric are carried out, and there is not only the splicing of large pieces but also the splicing of small pieces which allows small pieces to gather. Through this method the wonderful partial details of the clothing can be highlighted. Fourth, the hand-sewn decorative lines. The specific positions of the to-be-sewn decorative lines are firstly selected, and then the corresponding decorative lines are sewn according to the color of the fabric and the direction of the pattern (see "Fig. 7").

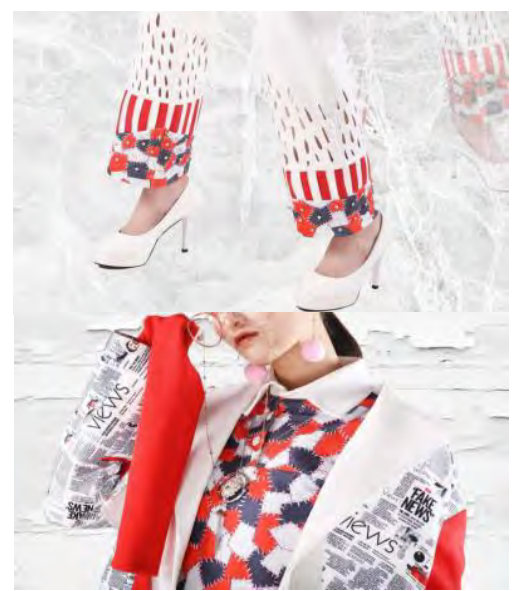

Fig. 7. Details of the hollowed, spliced parts and hand-sewn lines.

\section{Creative Modeling}

The creative silhouettes of the series of apparel include A and $\mathrm{H}$ shapes. The A shape is the shape that is narrower in the upper part and wider in the lower part. The round, foldable, high-necked collar is connected by the horn-like double-layered sleeves, forming the fake two-piece suit. The digitally printed patterns are distributed on the front and back of the body pieces, and the garment can be worn outside or inside. And a sleeveless jacket can be worn outside to increase the layers (as shown on the right side of "Fig. 8").
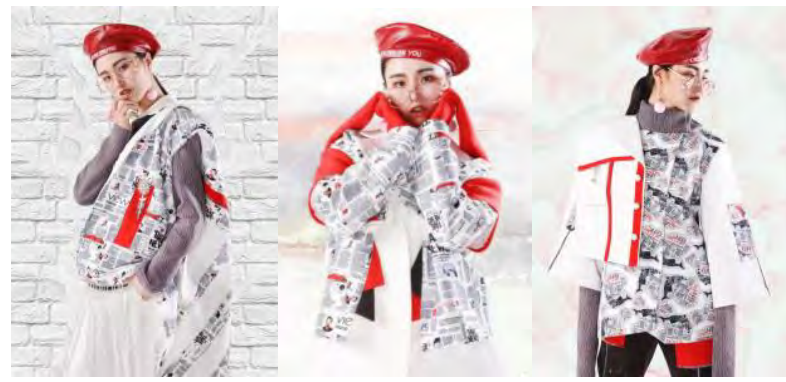

Fig. 8. The creative silhouettes of the series of apparel.

The H-shape is a straight shape with no waistline, as the plaid coat shown in the middle of "Fig. 8". The simple, vertical downward lines make the coat look sophisticated, casual and stable. The digitally printed patterns are distributed in the middle sleeves, front garments and back garments of the coat. The model wears this plaid coat with a pair of retro-style glasses and wears a beret which shows this season's resurgence of fashion, presenting a warm, chic and active personality. An element of the cotton rope is added to the bottom of the coat (as shown in the left side of "Fig. 9"), which adds a creative detail to the clothing. The cotton rope at the bottom flutters with the movement of the person, bringing a certain sense of agility to the coat and breaking the boring state of the whole suits.

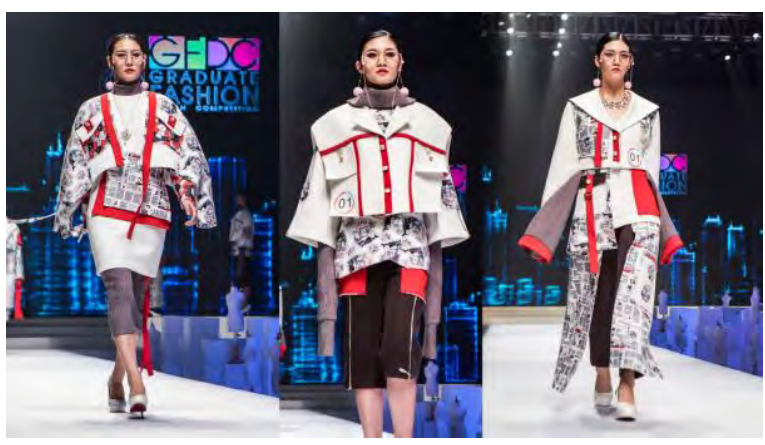

Fig. 9. Show Time Pictures.

The external form of the garment is closely related to the internal structure of it. A piece of clothing can be affected by different light, different angles, and different environments. However, the internal structure, color expression and fabric performance of the garment itself can directly determine the overall style of the garment.

\section{CONCLUSION}

In the design of this series of garments, I have accumulated many methods of fashion design and production. In the processes of extracting inspirations from market research materials, drafting garment renderings and style drawings, and producing the garments, I have explored a lot. First of all, the theory plays a guiding role in practice, and the true data from practice constantly denies the irrational designs and theoretical assumptions. The design of digital 
printing patterns and the layout of fabrics should be done in the early stage of digital printing. Finding the right type of fabric according to the style of the garment requires some consideration. Multiple trials must be made to find out the best effect of color expression for digital printing. Finally, the color expression with the best saturation is chosen for batch printing.

In the process of making the garment, the block surface of the fabric will be affected by the distribution of patterns. When cutting the fabric, it is necessary to reasonably consider the style of the garment, and the stitching relationship between the front and rear garments and the upper and lower garments should be especially paid attention to. The version of a piece of clothing is presented by multiple pieces, and each piece represents a part of the garment. The details also play a vital role in the design of the garment. Therefore, it is necessary to consider the connection between the block surface with designed patterns and the block surface with no patterns, and whether the connections of various parts can make the whole garment show a uniform and coordinated sense. In this process, we must not only take into account the details, but also highlight the main visual focus. The overall beauty of the garment, including the combination of color, fabric, process expression, innovative silhouette design, collocation, creative details, etc., requires a comprehensive consideration of the interaction and relationships between multiple elements. Among these elements, the fabric is the most important. The properties of the fabric determine the shape of the garment. In addition to considering the needs of the digital printing process, it is also necessary to consider the clothing style and degree of comfort of wearing the garment, so as to better display the ideal visual effect.

\section{REFERENCES}

[1] Journal of textile and apparel, technology and management Volume 4, Issue3, Spring 2005, 1st International Conference on Digital Technologies for the Textile Industries, 05 September 2013-06 September 2013, University of Manchester, UK. (In Press)

[2] Xie Chunping. Research on the application of digital printing patterns in clothing, Master's thesis of Jiang Nan university, 2013 (in Chinese)

[3] Chen Xin and Wu Pengpeng. Research on the application of digital printing textile fabrics in clothing design, Textile Guide, 2014 (in Chinese).

[4] Zhang Wenhui, Wang LiShi, Jin Yi. Detailed explanation of fashion design process, Dong Hua university press, 2014 (in Chinese).

[5] Bao Lei, Chen Xiaopeng. Application analysis of digital printing in clothing design, ShanDong textile Economy, 2015 (in Chinese).

[6] Shen Jingzhao. Fashion and Creativity - Research on the theory and method of fashion design, China Textile Press, 2017 (in Chinese).

[7] He Miao. Research on design and production of digital printing patterns, Art Technology, 2017 (in Chinese) 